\title{
A retrospective study on persistent pain after childbirth in the Netherlands
}

This article was published in the following Dove Press journal: Journal of Pain Research

12 Januray 2016

Number of times this article has been viewed

\author{
Rianne C Bijl',2 \\ Liv M Freeman ${ }^{2}$ \\ Philomeen TM Weijenborg ${ }^{3}$ \\ Johanna M Middeldorp ${ }^{2}$ \\ Albert Dahan' \\ Eveline LA van Dorp' \\ 'Department of Anesthesiology, \\ ${ }^{2}$ Department of Obstetrics, \\ ${ }^{3}$ Department of Gynecology, Leiden \\ University Medical Center, Leiden, \\ The Netherlands
}

\begin{abstract}
Reported prevalence rates of persistent postpartum pain (PPP) range from less than $1 \%$ to almost $20 \%$. The aim of this study was to examine the prevalence of PPP in a Dutch cohort and to evaluate a possible causal role for specific risk factors on the development of chronic pain after childbirth. A questionnaire was sent to 960 postpartum women approximately 2 years after delivery. Primary outcome was pain that arose from childbirth at follow-up, and secondary outcomes included quality of life (QoL) and Hospital Anxiety and Depression Scale scores. Tested risk factors included mode of labor analgesia, history of negative effect, history of chronic pain, delivery route, parity, and ethnicity. A total of 495 (51.6\%) women participated. At a mean time of 2.3 postpartum years, $7.3 \%$ of women reported any pain and $6.1 \%$ reported significant pain related to the delivery. Compared to spontaneous delivery, cesarean delivery provided protection against persistent pain (odds ratio, $0.12 ; 95 \% \mathrm{CI}, 0.01-0.63, P<0.05$ ). None of the other risk factors, including remifentanil use for labor pain, were of influence on the prevalence of persistent pain. Women with PPP experienced greater negative effects and had lower QoL scores compared to women without pain. In this cohort of Dutch patients, PPP is a serious problem with a great impact on the physical and mental health of women.
\end{abstract}

Keywords: chronic pain after childbirth, chronic pain, partus, labor analgesia, remifentanil, epidural analgesia, risk factors

\section{Introduction}

Both vaginal and cesarean delivery are associated with significant tissue damage. Vaginal deliveries are associated with uterine contractions, dilation of the cervix and lower uterine segment, stretching and compression of pelvic and perineal structures, inflammation of cervical tissue, and tears in the birth canal. ${ }^{1,2}$ During a cesarean section (CS), tissue damage is related to the skin incision, traction on abdominal muscles and nerves, and incision in the lower segment of the uterus. ${ }^{2}$ Multiple studies show that severe pain following surgery is linked to development of chronic or persistent pain. ${ }^{3,4}$ Pain following childbirth is not different in this respect. The reported prevalence of persistent postpartum pain (PPP) in women who gave birth either vaginally or by CS varies from less than $1 \%$ to almost $20 \% .^{1,2,5-9}$ Apart from the differences in study samples and methods to report pain, this large range might be explained by the fact that most previous studies did not discriminate between preexisting and new onset pain from delivery. ${ }^{5,7,9}$ In contrast, Eisenach et al $^{2}$ defined the primary outcome measure as pain which began during delivery at a location which could be attributed to the delivery (eg, pelvis, perineum, and abdomen). In their study, PPP after childbirth was relatively rare, with a prevalence of $1.8 \%$ at 6 months and $0.3 \%$ at 12 months. 
In this study, we examined the prevalence of PPP in a cohort of Dutch women. We surveyed women who had participated in the RAVEL (Remifentanil patient controlled analgesia versus epidural analgesia during labor) trial. ${ }^{10}$ This multicenter randomized controlled equivalence trial examined the effect of remifentanil patient-controlled analgesia (RPCA) vs epidural analgesia (EA) for labor pain. Approximately 2 years after delivery, the women were queried about the presence of PPP as well as for signs of anxiety and depression. Additionally, the women were asked to recollect pain at 3 months after childbirth (the end of maternity leave in the Netherlands) and at the first birthday of the child to get an impression of the course of PPP. Logistic regression models were constructed to estimate the role of specific risk factors in the development of PPP. An important risk factor for development of postoperative persistent pain is treatment with remifentanil during surgery. ${ }^{11,12}$ Extrapolating these data to the perinatal setting leads to the hypothesis that the use of RPCA for labor pain may be associated with more complaints of PPP following childbirth compared to no analgesic treatment or epidural labor analgesia.

\section{Methods}

\section{Study design and population}

The RAVEL trial was registered in the Dutch Trial Register (http://www.trialregister.nl) under number NTR2551. Women who had participated in that trial received a follow-up questionnaire in July $2014 .{ }^{10}$ In that trial, 1,414 parturients who received secondary or tertiary obstetric care (obstetric care at home was excluded) in one of 15 participating hospitals in the Netherlands were randomized to RPCA ( $30 \mu \mathrm{g}$ bolus with a lockout time of 3 minutes) or EA (either with ropivacaine/ sufentanil, bupivacaine/sufentanil, levobupivacaine/sufentanil, or bupivacaine/fentanyl), should they ask for pain relief during labor. The inclusion criteria for this follow-up study were participation in the RAVEL trial, age $>18$ years, and American Society of Anesthesiologists (ASA) physical status I or II. Women were excluded for follow-up if the written informed consent form was not present, if there was no permission to be contacted for follow-up study, if no contact information was available, or if the child had died. Women who delivered by primary CS were excluded in the analysis of the RAVEL trial, and therefore no data were available for this follow-up study. The study protocol was approved by the Leiden University Medical Center medical ethics committee.

\section{Data collection and outcome measures}

Women received an e-mail link to the Dutch Postpartum Chronic Pain Questionnaire (DPCPQ) or a paper version. The questionnaire was designed to assess pain complaints that began during delivery and were still present at three specific times after childbirth: end of maternity leave, first birthday of the child, and time of the survey. The DPCPQ was made up of questions from specific surveys including the McGill Pain Questionnaire and the Hospital Anxiety and Depression Score (HADS), ${ }^{13,14}$ as well as additional questions aimed at the presence of pain before and during pregnancy and the quality of life (QoL). The English translation of the DPCPQ is available from the authors. Since we had access to the database of the RAVEL trial, specific data regarding baseline patient and

Table I Baseline characteristics of the participating women

\begin{tabular}{|c|c|c|c|c|}
\hline & \multirow{2}{*}{$\begin{array}{l}\text { Complete } \\
\text { set }(n=495)\end{array}$} & \multicolumn{3}{|c|}{ Mode of labor analgesia } \\
\hline & & $\begin{array}{l}\text { None } \\
(n=199)\end{array}$ & $\begin{array}{l}\text { Remifentanil } \\
\text { PCA }(n=133)\end{array}$ & $\begin{array}{l}\text { Epidural } \\
\text { analgesia }(n=\mid 19)\end{array}$ \\
\hline Maternal age (years; mean $\pm \mathrm{SD})^{\mathrm{a}}$ & $34.6 \pm 4.5$ & $34.6 \pm 3.9$ & $4.4 \pm 5.0$ & $34.8 \pm 4.6$ \\
\hline Ethnic origin: Caucasian & 426 & 167 & 124 & 112 \\
\hline Higher education & 251 & 96 & 65 & 73 \\
\hline $\mathrm{BMI}\left(\mathrm{kg} / \mathrm{m}^{2} ; \text { mean } \pm \mathrm{SD}\right)^{\mathrm{b}}$ & $24.7 \pm 4.3$ & $24.4 \pm 4.4$ & $24.6 \pm 4.3$ & $25.3 \pm 4.2$ \\
\hline \multicolumn{5}{|l|}{ ASA classification } \\
\hline I & 328 & 128 & 98 & 87 \\
\hline 2 & 144 & 70 & 35 & 31 \\
\hline \multicolumn{5}{|l|}{ Parity ${ }^{c}$} \\
\hline 0 & 244 & 77 & 70 & 75 \\
\hline$\geq 1$ & 228 & $|2|^{c}$ & 63 & 43 \\
\hline Multiple pregnancy & 24 & $6^{c}$ & 7 & 10 \\
\hline Time to follow-up (years; mean $\pm S D$ ) & $2.3 \pm 0.3$ & $2.3 \pm 0.3$ & $2.3 \pm 0.3$ & $2.3 \pm 0.3$ \\
\hline
\end{tabular}

Notes: Data are shown as number unless otherwise indicated; age at time of the delivery; ${ }^{b} \mathrm{BMl}$ obtained at 3 months of pregnancy; ${ }^{\text {in }}$ the group of women who did not receive analgesia there were significantly more multiparous women $(P<0.00 \mathrm{I})$ and less women with a multiple pregnancy $(P=0.04 \mathrm{I})$ compared to the women who received analgesia.

Abbreviations: PCA, patient-controlled analgesia; BMI, body mass index; SD, standard deviation; ASA, American Society of Anesthesiologists. 
delivery characteristics were taken from the original RAVEL database. The following items were collected: pain at three postpartum time points, labor analgesia (RPCA, EA, or no analgesia), delivery route (spontaneous vaginal, instrumental vaginal, or CS), maternal age at delivery, body mass index (obtained at 3 months of pregnancy), ethnicity, ASA class, history of chronic pain, history of abdominal or back surgery, history of depression and mood disorders, parity, multiple pregnancy, the health-related QoL at the time of the survey (scored on a 10-point scale; question 18 of the DPCPQ), and anxiety and depression score at the time of the survey (derived from the HADS questionnaire). The HADS scores were divided into three subgroups: $0-7,8-10$, and 11-21 points, indicating absence, possible, and probable presence of anxiety or depression, respectively.

The primary outcome measures were 1) pain, defined as pain scored on an 11-point numerical rating scale (NRS) with a score $\geq 1$, which was present at the time of the survey and strictly localized at the lower back, abdomen, pelvis, vagina, or perianal area, and which began during delivery, and 2) significant pain, defined as pain as described above but with an NRS $\geq 3$ and/or which interfered with normal daily activities, including child care, housekeeping, or work. Secondary outcome measures were the health-related QoL, anxiety, and depression scores at the time of the survey.

\section{Statistical analysis}

Multivariate logistic regression was used to estimate the odds ratio (OR) for the occurrence of pain and significant pain at the time of the survey, adjusting for risk factors. To identify potential risk factors, univariate logistic regression models were fit to the data. Initial risk factors tested were type of labor analgesia, conversion from EA to RPCA or from RPCA to EA, history of depression and anxiety, history of chronic pain, history of back or abdominal surgery, delivery route, parity, and ethnicity. Those factors with a Wald test $P$-value of $<0.20$ were taken into the multivariable model. Two separate logistic models were constructed. The first was on the total population, and the second was on the women who received either EA or RPCA.

For secondary endpoints, differences in the QoL and HADS scores between patients without and with significant pain were assessed using $t$-tests. The HADS score before childbirth was compared to the recent score with a paired sample $t$-test.

SPSS version 20 (SPSS Inc., Chicago, IL, USA) was used to perform the statistical analysis. $P$-values $<0.05$ were considered significant.

\section{Results}

Of the 1,414 women enrolled in the RAVEL trial, 960 women did not object to participate in follow-up studies (Figure 1). The response rate was $51.6 \%(n=495$; last questionnaire received August 18, 2014). Of these 495 women, 346 had a spontaneous vaginal delivery, 42 an instrumental vaginal delivery, 95 a (secondary) CS, and there were 12 missing values. The number of women treated with RPCA and EA for labor pain was 133 and 119, respectively; 199 women received no pain relief. The remaining 44 women received intramuscular morphine, delivered by elective primary CS, or received both types of analgesia consecutively during labor. Table 1 gives the baseline characteristics of the parturients. In the group of women who did not receive analgesia, there were significantly more multiparous women $(P<0.001)$ and less women with a multiple pregnancy $(P=0.04)$ compared to the women who did receive analgesia. Other items did not differ between treatment groups.

\section{Prevalence of persistent pain following childbirth}

The mean time interval from delivery to the survey was 2.3 (SD: 0.3; range: 1.3-3.2 years). In Figure 2, the occurrence of PPP is given for the total population and for the three distinct treatment groups. At the time of the survey, 7.3\% (95\% confidence interval [CI], 5.1\%-9.9\%; $n=35$ ) of women reported any pain (NRS $>0$ ). The median NRS was 4.5 , with an interquartile range of 4.0. Pain frequency ranged from daily $(n=9)$ to near-daily pain (2-31 days without pain in the preceding month). There was no difference in demographics between women who developed pain and those who did not. Pain complaints were chiefly localized to the lower abdomen,

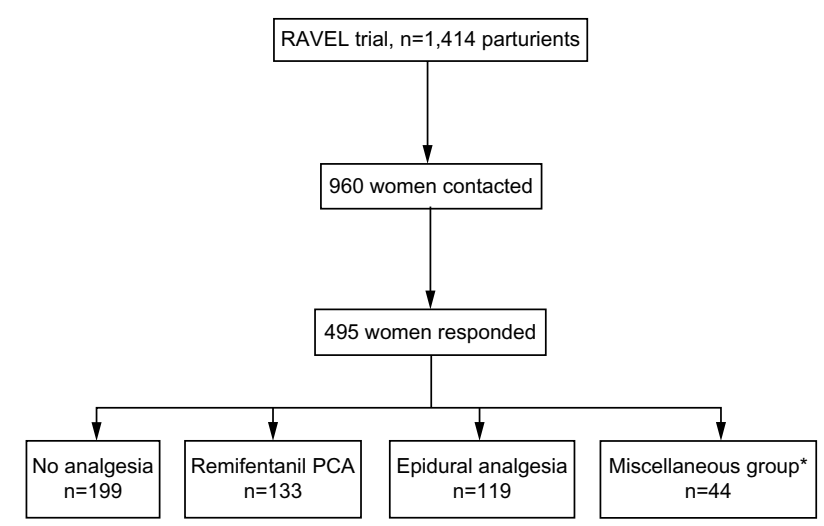

Figure I Flow chart of the study.

Note: *This group includes women who received intramuscular morphine for labor analgesia, delivered by elective primary cesarean section, or received both types of analgesia consecutively during labor.

Abbreviation: PCA, patient-controlled analgesia. 

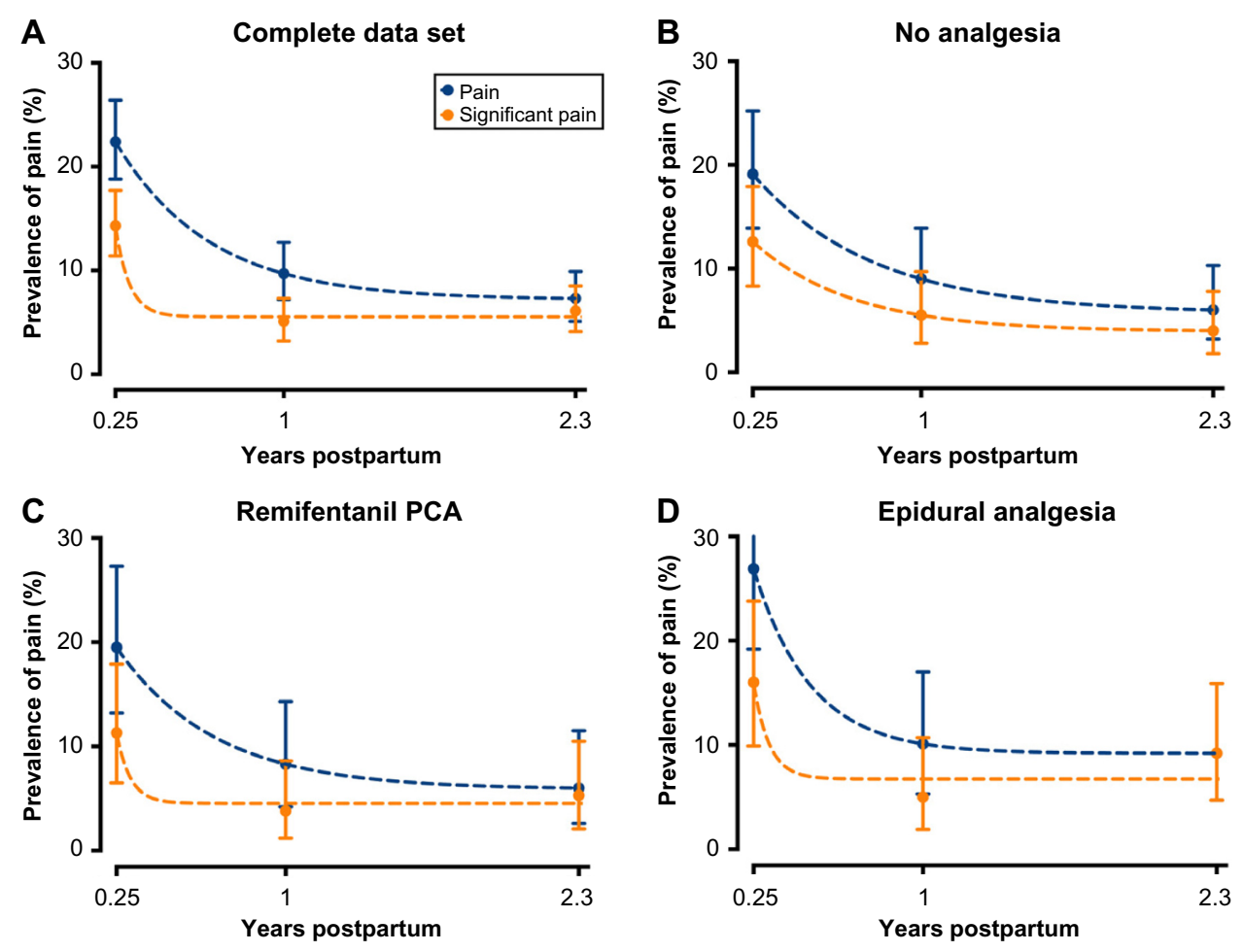

Figure 2 Pain that began during delivery and was located in the lower back, abdomen, pelvis, vagina, or perianal area reported at the time of the survey (on average 2.3 years following childbirth) and retrospectively at 3 months and I year following childbirth.

Notes: The data are shown for the total population (A) and women who received no analgesia during labor (B), women who received remifentanil PCA during labor (C) and women who received epidural analgesia during labor (D). Data are mean \pm upper and lower $95 \%$ confidence interval. Blue dots indicate all reported pain; orange dots indicate significant pain (NRS $\geq 3$ and/or pain which interfered with childcare, housekeeping, or work). To guide the eye, exponential functions are fit through the data.

Abbreviations: NRS, Numeric Rating Scale; PCA, patient-controlled analgesia.

pelvis, lower back, tailbone, vagina, perianal area, and/or groin. Thirty of these women $(6.1 \%, 4.1 \%-8.5 \%)$ indicated that the pain was significant with an NRS $\geq 3$ and pain affecting their daily activities (eg, child care, housekeeping, and/ or work); 23 had visited a doctor because of the pain and 13 required analgesia. In eleven women, the pain was such that it affected their sleep pattern.

Approximately 111 women reported PPP at the end of the maternity leave $(22.4 \%$; 95\% CI, $18.8 \%-26.4 \%) ; 71$ $(14.3 \%, 11.4 \%-17.7 \%)$ reported significant pain. Fortyeight women reported that they had PPP at the first birthday of their child $(9.7 \%, 7.2 \%-12.7 \%) ; 25(5.1 \%, 3.2 \%-7.3 \%)$ had significant pain.

Logistic regression analysis showed that various factors did not reach the cutoff level of $P<0.02$ in the univariate analysis, including history of depression and anxiety (negative effect), history of chronic pain, and history of back or abdominal surgery. The results of the analysis on the multivariate analysis of the complete data set are given in Table 2. Compared to women with no request for analgesia during labor, neither RPCA nor EA was a significant risk factor for PPP or significant PPP. Similarly, factor conversion, ethnicity, parity, and assisted vaginal delivery did not have influence on the prevalence of PPP. Women who delivered via CS were protected for development of PPP (one of 95 women; OR: 0.13, 95\% CI: 0.01-0.64, $P<0.05$ ) and significant PPP (one of 95 women; OR: $0.12,95 \%$ CI: $0.01-0.63, P<0.05)$ relative to women who spontaneously

Table 2 Output of multivariable logistic regression model performed on the complete data set of 495 women

\begin{tabular}{|c|c|c|}
\hline & \multicolumn{2}{|c|}{$\begin{array}{l}\text { Odds ratio ( } 95 \% \text { confidence } \\
\text { interval) }\end{array}$} \\
\hline & Pain & Significant pain \\
\hline $\begin{array}{l}\text { Use of remifentanil PCA during } \\
\text { labor vs no analgesia }\end{array}$ & $0.97(0.35-2.56)$ & $1.22(0.39-3.72)$ \\
\hline $\begin{array}{l}\text { Use of epidural analgesia during } \\
\text { labor vs no analgesia }\end{array}$ & $1.89(0.75-2.56)$ & $2.7 \mathrm{I}(0.99-7.74)$ \\
\hline Conversion vs no conversion ${ }^{\mathrm{a}}$ & $3.20(0.42-16.28)$ & $3.7 \mathrm{I}(0.50-18.2)$ \\
\hline $\begin{array}{l}\text { Ethnicity: Caucasian vs non- } \\
\text { Caucasian }\end{array}$ & $2.85(0.60-10.20)$ & $4.29(0.90-|5.5|)$ \\
\hline Parity: multiparous vs nulliparous & $1.89(0.83-4.55)$ & - \\
\hline $\begin{array}{l}\text { Assisted vs nonassisted vaginal } \\
\text { delivery }\end{array}$ & $2.00(0.61-5.69)$ & $1.40(0.40-4.10)$ \\
\hline $\begin{array}{l}\text { Cesarean section vs vaginal } \\
\text { spontaneous delivery }\end{array}$ & $0.13(0.01-0.64)^{*}$ & $0.12(0.01-0.63)^{*}$ \\
\hline
\end{tabular}

Notes: aConversion from remifentanil PCA to epidural analgesia or epidural analgesia to remifentanil PCA. The notation "-" denotes not included in the model. ${ }^{*} P<0.05$. Abbreviation: PCA, patient-controlled analgesia. 
delivered ( 29 and 23 of 346 women had pain and significant pain, respectively). There was no added pain prevalence in women exposed to remifentanil during labor compared to women who received EA, with an OR of 1.95 (95\% CI, $0.71-5.30 ; P>0.05)$ for pain and $0.45(95 \% \mathrm{CI}, 0.16-1.27$, $P>0.05$ ) for significant pain.

\section{QoL, anxiety, and depression following childbirth}

At an average of 2.3 years following childbirth, women with pain had a lower QoL score than women without pain: 7.0 [1.1] vs 7.8 [1.1], respectively $(P<0.001)$.

Mean anxiety scores in women with and without significant pain were 6.6 [4.1] and 4.6 [3.1] $(P=0.008)$, respectively. Approximately $19.4 \%(95 \% \mathrm{CI}, 8.2 \%-36.0 \%, \mathrm{n}=7)$ of women with PPP had probable anxiety (HADS score $11-21)$ vs $5.1 \%(3.2 \%-7.5 \%, n=23)$ of women without pain. Approximately $27.7 \%(14.2 \%-45.2 \%, \mathrm{n}=10)$ of women with PPP had possible (score 7-10) or probable anxiety vs $16.5 \%$ $(13.2 \%-20.3 \%, n=75)$ of women without pain (Figure 3).

Mean depression scores in women with and without significant PPP were $4.6 \pm 3.8$ and 2.6 $\pm 2.8(P=0.004)$, respectively.
Approximately 5.6\% (95\% CI, $0.7 \%-18.7 \%, \mathrm{n}=2)$ of women with PPP had probable depression (HADS score 11-21) vs $1.8 \%(0.8 \%-3.4 \%, \mathrm{n}=8)$ of women without pain; $16.7 \%$ $(6.4 \%-32.8 \%, n=6)$ of women with PPP had possible (score $7-10)$ or probable depression vs $7.9 \%(5.6 \%-10.8 \%, n=36)$ without pain (Figure 3).

There were no differences in prenatal HADS scores between women who would develop PPP and who would not, with mean scores for anxiety 4.9 [2.4] (significant pain) and 4.5 [2.9] (no pain, $P=0.516$ ) and mean scores for depression 3.5 [2.8] (significant pain) and 2.7 [2.5] (no pain, $P=0.116$ ), respectively (Figure 3 ).

\section{Discussion}

Taken the many millions of births each year, the occurrence of PPP that interferes with the daily activities in even a small percentage of women will have a large socioeconomic impact. In our cohort of 495 Dutch women, significant pain attributed to the delivery process was present in $6.1 \%$ (any pain in $7.3 \%$ ) of women, on average 2.3 years after childbirth. Extrapolation of these findings to the 170,000 deliveries per year in the Netherlands suggests that approximately
A Anxiety: before vs after childbirth

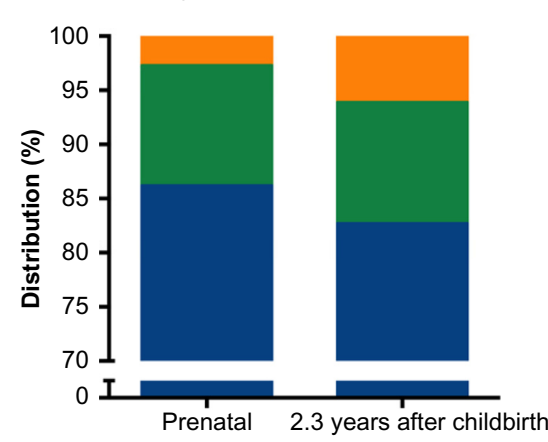

C Depression: before vs after childbirth

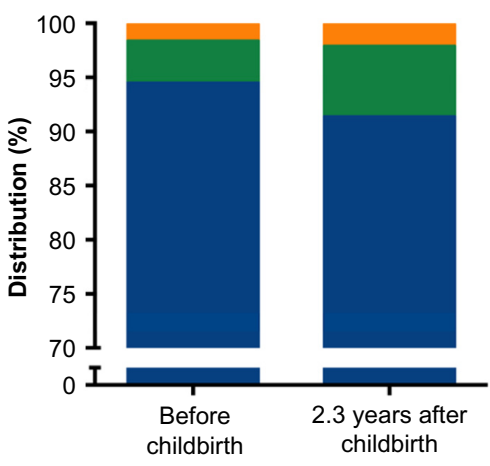

B Anxiety at 2.3 years: pain vs no pain

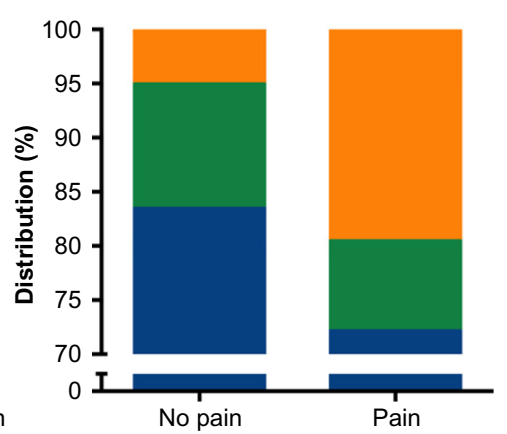

D Depression at 2.3 years: pain vs no pain

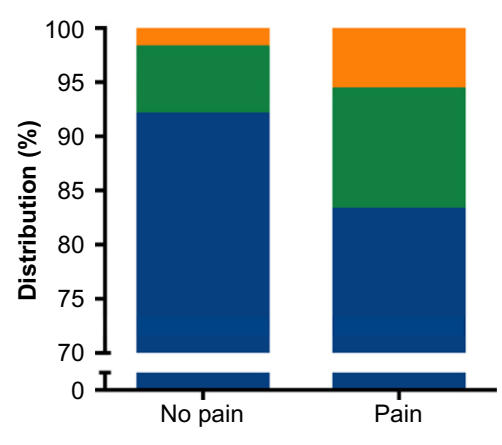

HADS score 0-7

HADS score 8-10

HADS score 11-21

Figure 3 Distribution of HADS scores for anxiety and depression.

Notes: (A) Anxiety scores: before delivery vs on average 2.3 years after delivery. (B) Anxiety scores in women without vs with pain. (C) Depression scores: before vs on average 2.3 years after delivery. (D) Depression scores in women without vs with pain. HADS scores 0-7, 8-10, and II-2I points indicate absence, possible, and probable presence of anxiety or depression, respectively.

Abbreviation: HADS, Hospital Anxiety and Depression Scale. 
10,000 women are affected yearly with significant pain. ${ }^{15}$ The presence of persistent pain was associated with a reduced health-related QoL and a fourfold higher prevalence of anxiety symptoms and threefold higher prevalence of negative-effect-related symptoms compared to women without pain. A protective effect was observed following CS, while none of the other risk factors influenced the prevalence of PPP. Importantly, treatment of labor pain with either EA or RPCA had no effect on the occurrence of pain. Retrospective questions in our survey revealed that the prevalence of significant pain at the end of maternity leave was $14.3 \%$ (any pain $22.4 \%$ ) and at the child's first birthday was $5.1 \%$ (any pain $9.7 \%$ ), which indicates that no resolution occurred in the second year of postpartum pain.

The questions in the DPCPQ were such that the women were forced to restrict pain reporting to symptoms that arose during delivery (ie, "new" pain). The 95\% lower limit of significant pain prevalence at the time of the survey was $4.1 \%$. The response rate of our survey $(-52 \%)$ was relatively low and may have caused an outcome bias. One possibility is that the women who did not respond were without any pain complaints at the time of the survey. Taking this assumption into account, the prevalence of significant pain might be as low as $3.1 \%$ with a lower limit of $2.1 \%$. This is still considerably higher than the recently reported prevalence rate in the study by Eisenach et al, ${ }^{2}$ who showed that women in the United States have a PPP prevalence of $0.3 \%$ at 1 -year postdelivery. As indicated previously, Eisenach et $\mathrm{al}^{2}$ also restricted the analysis to "new" pain. Apart from the evident differences in protocols (the US study was a prospective longitudinal cohort trial in two tertiary centers in North Carolina and New York), specific circumstances surrounding the delivery process may differ. Eisenach et $\mathrm{al}^{6}$ showed further that the severity of acute pain after delivery was associated with the risk of transition to chronic pain. This would suggest that women in the Dutch cohort experienced more severe acute pain after delivery than women in the USA. We did not query the women regarding immediate postpartum pain. The first interaction of the mother with her newborn child is highly emotional and we contend that this may have affected the mothers' recollection at that moment in time. Still, there are no reasons to assume any differences in intra- and postpartum care between the two countries, and hence, any difference in acute postpartum pain between studies. Another possibility for the difference in outcome may be related to the number of CSs. The number of CSs was greater in the US cohort (32\% vs $19 \%){ }^{2}$ Still in the US study, the mode of delivery did not represent a significant risk factor for chronic pain development, and in our study women who delivered predominantly by secondary CS were relatively protected from PPP development. Alternatively, and possibly most importantly, there may be biopsychosocial differences surrounding delivery that may play a causal role in the difference in PPP development between the two cohorts (eg, factors related to endogenous modulation of pain, pain catastrophizing, coping strategies, genetics, etc). Future prospective studies should address which risk factors for chronic pain are responsible for the relatively high PPP rates observed in the Netherlands.

The survey was taken on average 2.3 years after delivery. Since the RAVEL trial was not designed to study PPP, we had to rely on retrospective questions to assess pain complaints at 3 months and 1 year after childbirth, introducing a possible recall bias. It is likely that women who experienced pain at the time that they received the DPCPQ read and pondered the retrospective questions more thoroughly than the women without pain. However, by focusing on two time points, which most probably had a rather large impact on the women's life (end of maternity leave and first birthday of the child), we believe that we created two windows of increased awareness regarding possible pain symptoms. Consequently, we contend that this may have increased the reliability of answers, also in women without pain at the time of the survey. We relate the small increase in significant pain from 1 to on average 2.3 years after delivery (from $5.1 \%$ to $6.1 \%$, Figure $2 \mathrm{~A}$ ), apart from the obvious recall bias, to the possibility that chronic pain is not a continuous symptom but fluctuates over time in terms of severity. These data suggest that chronic postpartum pain resolution more than 1 year following childbirth is tedious. In future surveys, we will further monitor the course of pain in our cohort.

We had to reject our hypothesis that, in common with intraoperative use of remifentanil, obstetric RPCA increases the prevalence of PPP. Although we corrected for possible confounders, the study may have been underpowered to detect a remifentanil effect. Another explanation may be that the remifentanil dose is an important factor in the development of chronic pain. The mean remifentanil dose in our study was $1.49 \mathrm{mg}$; much higher doses are given during anesthesia. This reasoning is supported by the reported dose-dependent impact of remifentanil analgesia on the development of chronic thoracic pain after sternotomy. ${ }^{12}$

Somewhat unexpectedly, CS protected the women from PPP compared to spontaneous vaginal delivery, with just 
one woman (out of 95) with PPP following CS (OR 0.12 for pain and 0.13 for significant pain, Table 1). In most women, CS was performed under spinal or epidural anesthesia. For postoperative analgesia, paracetamol, opioids (eg, methadone or morphine), and nonsteroidal anti-inflammatory drugs were given in case of CS. As discussed by Eisenach et al, ${ }^{2}$ it is reasonable to assume that tissue injuries during surgery for a CS, such as traction on abdominal structures and nerves (eg, the ilio-inguinal and hypogastric nerves) and damage to the lower uterine segment, would increase the likelihood of PPP. In our cohort, most women who delivered by CS had contractions and some degree of cervical dilation (secondary CS occurred in 84 of 95 women), adding to the degree of tissue injury that could cause persistent pain. It is plausible that the protective effect of CS is related to the postoperative treatment of pain with opioid and/or EA. This reasoning is in agreement with the theory that severe postpartum pain is associated with a high probability of development of PPP, and suggests that effective relief of severe pain after vaginal delivery, for example by EA, would reduce the prevalence of PPP. ${ }^{1}$

We established once more that PPP and negative effect are comorbid. ${ }^{16}$ While prenatal anxiety and depression scores were not different between women who developed PPP and those who did not, at the time of the survey the differences in HADS were significant with greater anxiety and depression scores and a reduced QoL in women with PPP compared to those without pain (Figure 3). This exemplifies the impact of chronic pain on the mental health of this relatively young population. Interestingly, we did not find a correlation between negative effect before delivery and the development of PPP. While for the transition from acute postsurgical pain to persistent pain this correlation is well established, ${ }^{17-19}$ this association is less apparent for PPP. ${ }^{2,7}$

The patient population that was targeted in our study included women in secondary and tertiary obstetric care. Since more than $20 \%$ of pregnant women in the Netherlands give birth in primary, midwife-led care, our results do not apply to the total population of Dutch pregnant women. Development of chronic pain in primary obstetric care remains unknown at present. Other limitations of the study included the relatively low response rate $(52 \%)$ and the retrospective nature of our study. Our study differs from most previous studies in that it, in contrast to our current study, did not make a distinction between pain complaints specifically related to childbirth and preexistent pain. Taken together, we contend that despite some limitations our study shows that persistent significant "new" pain following childbirth in this Dutch cohort is a serious problem with a relatively high prevalence at 2 years postpartum.

\section{Conclusion}

We retrospectively surveyed women on the prevalence of persistent pain following childbirth that began during delivery. We observed that in 495 women, at a mean time of 2.3 postpartum years, $6.1 \%$ complained of significant pain related to delivery. Compared to spontaneous delivery, cesarean delivery provided protection against persistent pain. Our results further show important implications for the physical and mental health of the women in pain.

\section{Acknowledgment}

This study was funded by a grant from ZonMW (Dutch Organization for Health Care Research and Development) project number 80-82310-97-11039.

\section{Author contributions}

All authors contributed toward protocol writing, data acquisition, data analysis, drafting and critically revising the paper, gave final approval of the version to be published, and agree to be accountable for all aspects of the work.

\section{Disclosure}

The authors report no conflicts of interest in this work.

\section{References}

1. Vermelis JM, Wassen MM, Fiddelers AA, Nijhuis JG, Marcus MA Prevalence and predictors of chronic pain after labor and delivery. Curr Opin Anaesthesiol. 2010;23:295-299.

2. Eisenach JC, Pan P, Smiley RM, et al. Resolution of pain after childbirth. Anesthesiology. 2013;118:143-151.

3. Kehlet $\mathrm{H}$, Jensen TS, Woolf CJ. Persistent postsurgical pain: risk factors and prevention. Lancet. 2006;367:1618-1625.

4. Schug SA, Pogatzki-Zahn EM. Chronic pain after surgery or injury. Pain Clin Updates (IASP). 2011;19:1-5.

5. Howell CJ, Dean T, Lucking L, et al. Randomised study of long term outcome after epidural versus non-epidural analgesia during labour. BMJ. 2002;325:357-360.

6. Eisenach JC, Pan PH, Smiley R, et al. Severity of acute pain after childbirth, but not type of delivery, predicts persistent pain and postpartum depression. Pain. 2008;140:87-94.

7. Kainu JP, Sarvela J, Tiippana E, et al. Persistent pain after caesarean section and vaginal birth: a cohort study. Int J Obstet Anesth. 2010;19: 4-9.

8. Landau R, Bollag L, Ortner C. Chronic pain after childbirth. Int J Obstet Anesth. 2013;22:133-145.

9. Nikolajsen L, Sorensen HC, Jensen TS, Kehlet H. Chronic pain following Caesarean section. Acta Anaesthesiol Scand. 2004;48:111-116.

10. Freeman L, Bloemenkamp W, Franssen MT, et al. Remifentanil patient controlled analgesia versus epidural analgesia in labour; a randomised multicentre equivalence trial. BMJ. 2015;350:h846. 
11. Salengros JC, Huybrechts I, Ducart A, et al. Different anesthetic techniques associated with different incidences of chronic post-thoracotomy pain: low-dose remifentanil plus presurgical epidural analgesia is preferable to high-dose remifentanil with postsurgical epidural analgesia. $J$ Cardiothorac Vasc Anesth. 2010;24:608-616.

12. van Gulik L, Ahlers SJ, van de Garde EM, et al. Remifentanil during cardiac surgery is associated with chronic thoracic pain 1 year after sternotomy. Br J Anaesth. 2012;109:616-622.

13. Vanderiet K, Adriaensen H, Carton H, Vertommen H. The McGill Pain Questionnaire constructed for the Dutch language (MPQ-DV). Preliminary data concerning reliability and validity. Pain. 1987;30:395-408.

14. Zigmond AS, Snaith RP. The hospital anxiety and depression scale. Acta Psychiatr Scand. 1983;67:361-370.

15. StatLine; Birth; key figures [September 18 2015]. Statistics Netherlands. Available at http://statline.cbs.nl/Statweb/publication/?DM=SLEN\&PA= 37422eng\&D1 $=0-18,20,22-34,43-46,48 \& D 2=0,10,20,30,40,50,60,63-$ 64\&LA=EN\&VW=T. Accessed December 16, 2015.
16. Dahan A, van Velzen M, Niesters M. Comorbidities and the complexities of chronic pain. Anesthesiology. 2014;121:675-677.

17. Theunissen M, Peters ML, Bruce J, et al. Preoperative anxiety and catastrophizing: a systematic review and meta-analysis of the association with chronic postsurgical pain. Clin J Pain. 2012;28:819-841.

18. Connelly M, Fulmer RD, Prohaska J, et al. Predictors of postoperative pain trajectories in adolescent idiopathic scoliosis. Spine. 2014;39: E174-E181.

19. Dualé C, Ouchchane L, Schoeffler P, Dubray C; the EDONS Investigating Group. Neuropathic aspects of persistent postsurgical pain: a French multicenter survey with a 6-month prospective follow-up. J Pain. 2014;15:24. e1-24. e20.

\section{Publish your work in this journal}

The Journal of Pain Research is an international, peer-reviewed, open access, online journal that welcomes laboratory and clinical findings in the fields of pain research and the prevention and management of pain. Original research, reviews, symposium reports, hypothesis formation and commentaries are all considered for publication.

\section{Dovepress}

The manuscript management system is completely online and includes a very quick and fair peer-review system, which is all easy to use. Visit http://www.dovepress.com/testimonials.php to read real quotes from published authors. 